\title{
A Quantitative Description of Lipid and Extracellular Matrix Proteinaceous Fibers in Hepatic Fibrosis of a Rat Model by ImageJ using Nano-Images
}

Abdel-Majeed Safer*

Department of Biological Sciences, and Head of Nanoscopy Science Center, Faculty of Science, Kuwait University, P.O. Box 5969, Safat, 13060, Kuwait

\begin{abstract}
We made a histological and ultrastructural studies using Image J freeware and Origin pro statistical analysis of the site of lipid accumulations and ECM pertinacious collagen fibers in a rat model of hepatic fibrosis using $\mathrm{CCl}_{4}$ and ethanol dual effect and using nano GTE as the main factor to diminishes both hepatic lipid and ECM fibers. This imageJ observation indicates and re-enforces the notion previously published by the same author that Nano GTE is a strong therapeutic agent to work against the oxidative effects of chemicals like $\mathrm{CCl}_{4}$ and ethanol.
\end{abstract}

Keywords: Lipid; Fibber; Chitosan nano-green tea; Bio-nano particle; Hepatocytes; Image)

\section{Introduction}

It is now recognized that in the hepatofibrotic liver, lipids and fibrous proteins are known as steatosis and fibrosis respectively are enormous in liver parenchyma [1-4]. In several of our recently published works, although we have managed to establish their presence as an outcome of liver fibrosis and how nanogreen tea extract managed to remediate it in several technics $[4,5]$ yet the analysis of their features in tissues often remains qualitative. Thus, quantitative microscopy technique for imaging localized lipid and ECM fibers environments and measuring them in fixed tissues is needed to be explored.

This approach directly examines the organization of lipid and fibers. Image analysis tools have been developed to quantify such features, but they often involve an image processing stage that may bias the output and/or require specific software.

This paper aims at confirming once again what have been published earlier [5] about lipid accumulation and ECM fiber formation during the process of hepatic fibrosis by adopting combined ImageJ and Origin Pro 2017 for statistical Analysis [5-7]. These two Freeware combined for the first time - in analyzing nanoscopy images obtained from fibrotic group and group treated with nanogreen tea extract providing this new tool for exploring and re-enforcing the presence of the two district features that occurred during the various stages of hepatocirrhosis; lipid bodies and extracellular matrix fibers and how nanogreen tea extract remediates both [4].

\section{Materials and Methods}

\section{Experiments}

Preparation of chitosan nano-GTE: Preparation of green tea extract (GTE) and encapsulation of GTE within chitosan nanoparticles (including characterization methods) to make chitosan nano-GTE was done as described in our earlier paper [4].

Animals: Forty male Sprague-Dawley rats weighing between 200$250 \mathrm{gms}$ were used in this study. All animals were obtained and housed in the Animal House, Department of Biological Sciences, Kuwait University. The Animal House is a well-established facility with an International Code of Ethics. The rats were kept in plastic cages in a controlled environment of $40 \%$ humidity at $22^{\circ} \mathrm{C}$, with $12 \mathrm{hr}$ light/dark period and treated gently. All rats had free access to tap water ad libitum and pellet rodents chow (SDS, Witham, UK). The composition of the diet was $14.7 \%$ protein, $2.6 \%$ fat, and $5.3 \%$ cellulose. Rats were divided into 4 groups (10 in each group) based on different oral treatments administered to them. Subcutaneous doses of $40 \% \mathrm{CCl}_{4}$ and oral doses of $25 \%$ ethanol, chitosan, and chitosan nano-GTE were administered to the animals as detailed in Table 1.

Tissue preparation for LM and SEM: Rats from each group were carefully anesthetized with ether followed by dissection of the abdominal cavity to expose the liver. The liver was immediately soaked with cold $2.5 \%$ glutaraldehyde/sodium cacodylate fixative, $\mathrm{pH} 7.2$, kept at $0-4^{\circ} \mathrm{C}$ for 2 hours then changed to a fresh fixative and left overnight. Tissues were then transferred to sodium cacodylate/sucrose buffer, 3 times for 20 minutes each, then transferred to $1 \% \mathrm{OsO}_{4} / \mathrm{PO}_{4}$ buffer for 2 hours and blocked in Epon.

Semi-thin sections were cut using Leica ultra-microtome for toluidine blue staining and photography.

For SEM, critical point drying (CPD) was used. The blocks were fixed on stubs and put in the sputter coater for shadowing the specimen with platinum/gold. Investigation and image acquisition were done on the Variable Pressure Field Emission remotely operable scanning electron microscope (Leo Supra 50VP) operated at $30 \mathrm{kV}$.

\section{Computational image analysis}

Hepatic lipids and collagen fibers were measured using ImageJ Freeware version V 1.51A plug-ins, downloaded from the NIH website (http://rsb.info.nih.gov/ij) were used (Scheme 1). ImageJ Freeware $\mathrm{V} 1.51 \mathrm{~A}$ is a public domain, image processing program developed at

\begin{tabular}{|c|c|c|c|}
\hline Label & Tissue Total Area & lipid Area (Pixel) & Area (\%) \\
\hline control (Green) & 3117876 & 100749 & 3 \\
\hline eth+ccl (Green) & 3145720 & 768008 & 28 \\
\hline eth+ccl + +gt (Green) & 3145728 & 121917 & 20 \\
\hline
\end{tabular}

Table 1: Quantification of the main tissue area and quantification of lipid and percentage.

*Corresponding author: Abdel-Majeed Safer, Department of Biological Sciences, and Head of Nanoscopy Science Center, Faculty of Science, Kuwait University, P.O. Box 5969, Safat, 13060, Kuwait, Tel: 96524985909; E-mail: amsafer.79@ku.edu.kw

Received: June 16, 2017; Accepted: June 23, 2017; Published: June 28, 2017

Citation: Safer AM (2017) A Quantitative Description of Lipid and Extracellular Matrix Proteinaceous Fibers in Hepatic Fibrosis of a Rat Model by ImageJ using Nano-Images. J Nanomed Nanotechnol 8: 446. doi: 10.4172/2157-7439.1000446

Copyright: ( 2017 Safer AM. This is an open-access article distributed under the terms of the Creative Commons Attribution License, which permits unrestricted use, distribution, and reproduction in any medium, provided the original author and source are credited. 


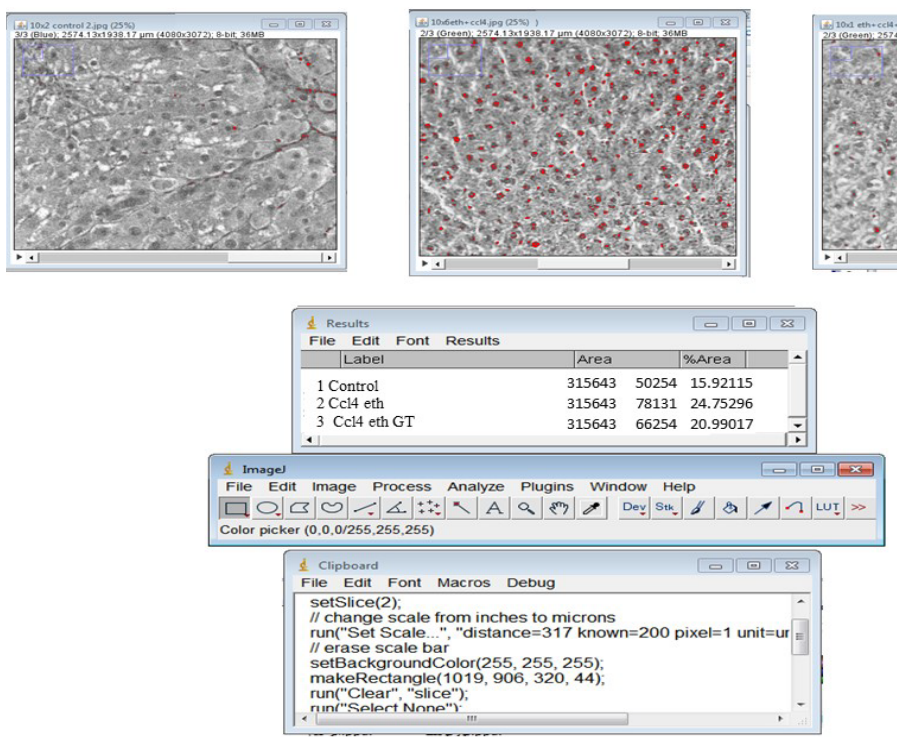

Scheme 1: Shortcut screen from imageJ software for Collagen fiber and macro program analysis.

the National Institutes of Health NIH. http://rsb.info.nih.gov/ij; It is a public domain Java image processing program inspired by NIH, USA [6-8].

\section{Quantification of hepatic lipid content before and after fibrosis}

Computational image analysis for the determination of the damaged regions of the tissue after the effect of CC4 and ethanol and the treatment with nanoGTE was carried out primarily with the this software package. Quantifications for the damaged, undamaged, and complete area of the tissue were carried out in pixels and the results were expressed in percentage of the ratio of the damaged to the complete tissue area [5]. To quantify the artificially made red-stained collagen in an image of a rat liver tissue section stained with $\mathrm{H} / \mathrm{E}$ and/or Masson's Trichrome stains Table 2. The following four main steps have been followed:

I. Change the scale to Nanometer,

II. Convert the image to grayscale,

III. Segment (isolate) the red-stained collagen using thresholding,

IV. Measure the thresholded area.

We can fix this by measuring the length of the scale bar and using the Analyze>Set Scale command from the main menu program to set the scale to nanometers. To measure the scale bar, we used the straight line selection tool (a fifth tool from the left on the ImageJ toolbar) to create a line selection along the length of the scale bar as shown below in short-cut of the screen [6-8].

Open the analyze: Set Scale dialog box. Notice that the length of the line selection (317 pixels) is automatically entered as the "Distance in Pixels". Enter the scale bar length $(200 \mu \mathrm{m})$ as the "Known Distance" and "um" as the "Unit of Length". ImageJ will automatically convert the "u" to the micrometer symbol $(\mu)$. Click "OK" and notice that the image size has changed to $858.95 \times 646.74 \mu \mathrm{m}[6,7]$.

Next use the image: $>$ Type $>$ RGB Stack command to split the image into red, green and blue channels. This is necessary because the Image $>$ Adjust $>$ Threshold tool only works with grayscale images. Try

\begin{tabular}{|c|c|c|c|}
\hline Label & Tissue Total Area & lipid Area (Pixel) & Area (\%) \\
\hline control (Green) & 315643 & 100749 & 15.9 \\
\hline eth+ccl (Green) & 315643 & 768008 & 44.7 \\
\hline eth+ccl + +gt (Green) & 315643 & 121917 & 21 \\
\hline
\end{tabular}

Table 2: Quantification of the main tissue area and quantification of fiber and percentage.

to threshold an RGB image and you will get a lot beeps and the status bar message "RGB images cannot be thresholded". Move the slider to view each of the channels. Notice that the green channel has the best separation $[7,8]$.

Use the Image $>$ Stacks $>$ Make Montage command to view all three channels at the same time: Select the RGB stack (with the Green channel selected) and press shift-t (Image $>$ Adjust $>$ Threshold). The "Threshold" tool opens and the green channel is automatically thresholded. Unfortunately, ImageJ is not able to correctly threshold this image so the threshold must be manually adjusted. Do this by moving the lower slider until the stained collagen is highlighted in red. Notice that the threshold level arrived at manually is about half the automatically set level of 175 . Click on "Set" and set the upper level to $175 / 2$, or 87 . Setting a threshold that is a fraction of the automatically determined threshold will allow us to later create a macro that does not require manual thresholding [9].

Next we must erase the scale bar to prevent it from being included in the calculated area: To do this, use the rectangular selection tool to select the scale bar, then press Backspace (Delete on the Mac). If the current background is not white, use the color picker tool (Image $>$ Color $>$ Color Picker) to change it to white. Note that the current background color is indicated by the color of the border surrounding the eye dropper tool icon [7].

We are almost ready, but first we have to tell ImageJ what to measure by opening the Analyze >Set Measurements dialog and checking "Area", "Area Fraction", "Limit to Threshold" and "Display Label". Then press "m" (Analyse >Measure) and the area and percent area will be displayed in the "Results" window. Right click in the "Results" window and select 
Citation: Safer AM (2017) A Quantitative Description of Lipid and Extracellular Matrix Proteinaceous Fibers in Hepatic Fibrosis of a Rat Model by Image J using Nano-Images. J Nanomed Nanotechnol 8: 446. doi: 10.4172/2157-7439.1000446

"Save As" to save the results as a tab-delimited text file or select "Copy" to copy the results to the Clipboard $[6,8]$.

Another way to measure the red-stained collagen is to convert the thresholded portion of the image to a selection using Edit $>$ Selecton $>$ Create Selection, transfer the selection to the original RGB images by selecting it and pressing shift-e (Edit $>$ Selecton $>$ Restore Selection), and then pressing "m" (Analyse $>$ Measure) to measure the area of the selection Table 3 [8].

\section{Quantification of hepatic collagen content before and after fibrosis}

Below is a macro that automates the analysis. To run it, copy it to the clipboard, switch to ImageJ, press shift-v (File\&gt;New\&gt;System Clipboard), click on the quot;Clipboard\&quot; window and press ctrl-r (Macros\&gt;Run Macro). Information about the ImageJ macro language is available at rsb.info.nih.gov/ij/developer/macro/macros. html [6-10]

\section{Macro program}

//select the green channel, which has the best contrast

$\operatorname{run}(\& q u o t ; R G B$ Stack\&quot;);

setSlice(2);

//change scale from inches to microns

$\operatorname{run}(\& q u o t ;$ Set Scale...\&quot; $\quad \& q u o t ;$ distance $=317$ known $=200$ pixel=1 unit=um\&quot;);

\begin{tabular}{|c|c|c|c|}
\hline Component & Control & $\mathbf{C C l}_{4}$ +ethanol & $\mathbf{C C l}_{\mathbf{4}}$ +ethanol+GT \\
\hline Lipid & $3 \%$ & $28 \%$ & $20 \%$ \\
\hline Fiber & $15 \%$ & $49 \%$ & $22 \%$ \\
\hline
\end{tabular}

Table 3: Quantification Percentage of fiber and lipid in different group. //erase scale bar

setBackgroundColor (255, 255, 255);

makeRectangle(1019, 906, 320, 44);

run(\&quot;Clear\&quot; \&quot;slice\&quot;);

$\operatorname{run}(\& q u o t ;$ Select None\&quot;);

//set threshold

setAutoThreshold();

getThreshold(min, max)

setThreshold(0, $\max / 2)$;

$/ /$ measure area and area fraction

run $(\& q u o t$;et Measurements...\&quot; \&quot;area area_fraction limit display redirect $=$ None

decimal=3\&quot;);

run(\&quot;Measure\&quot;);

selectWindow(\&quot;Results\&quot;);

A second image is available for testing the macro. The \& quot; Results \& quot; window below contains the output resulting from running the macro on both images. The \& quot; Label \& quot; column displays the image title and channel [11].

\section{Results}

\section{Lipid analysis}

Once threshold was set at a proper scale, from normal control (Figure 1a), all lipid droplets in the damaged tissue under study due to

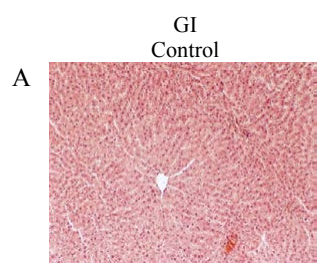

$\mathrm{D}$
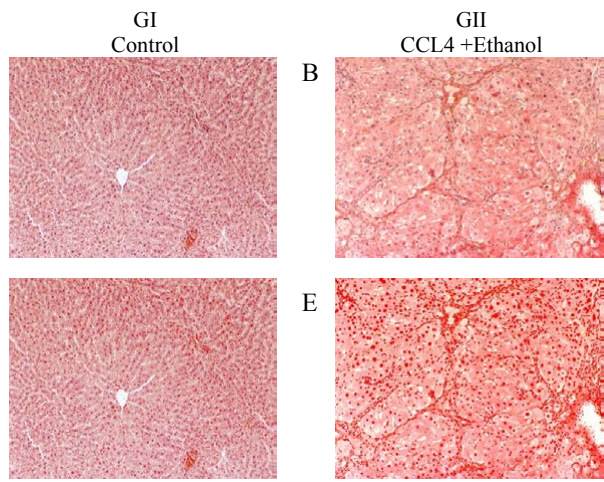

$\mathrm{E}$

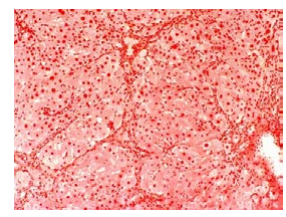

G

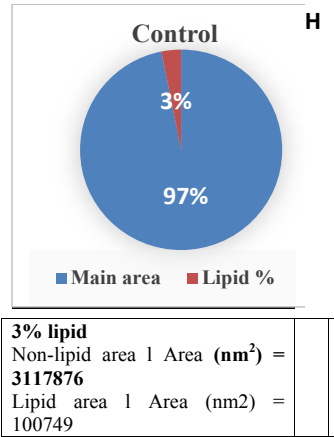

00749
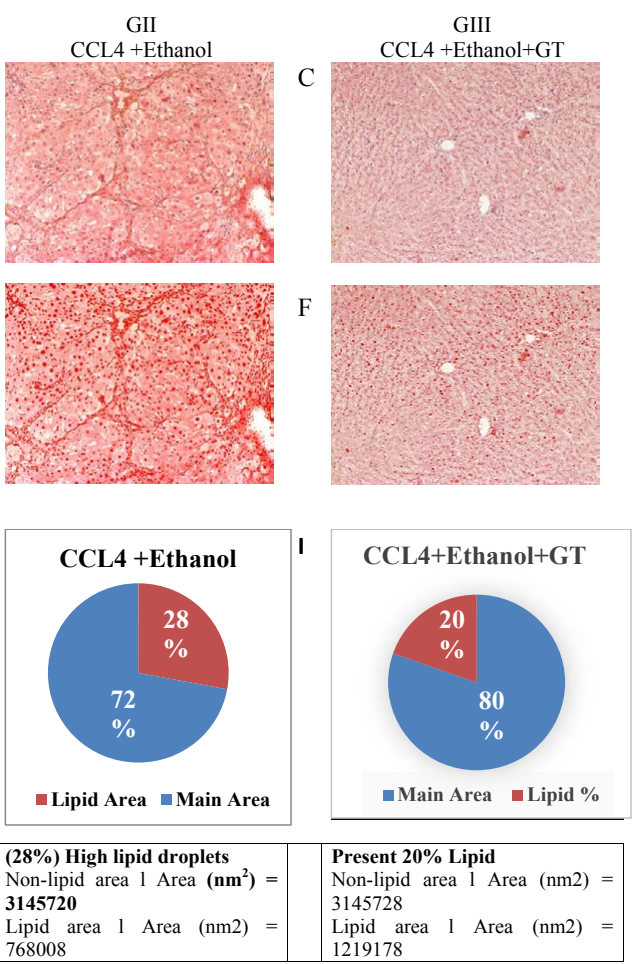

219178

Figure 1: Quantification of lipid comparison with different group Protein fiber analysis. 


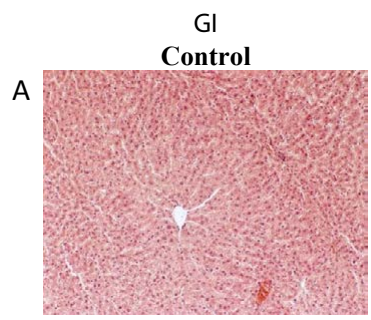

$\mathrm{D}$

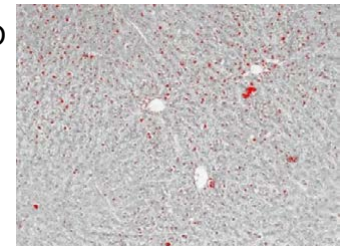

\section{9\% Fiber}

Tissue Area $=315643$

Fiber area $=50254$

G

50.0

45.0

40.0

35.0

30.0

25.0

20.0

15.0

10.0

5.0

0.0

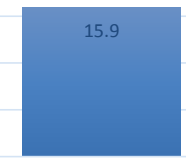

control
GII CCL4 +Ethanol

B

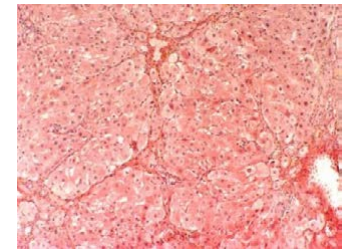

E

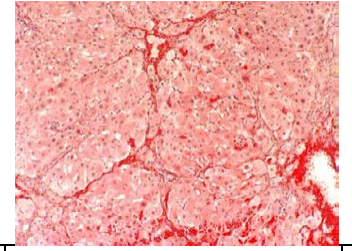

24.8\% Fiber droplets

Tissue Area $=315643$

Fiber area $=78131$
GIII

C CCL4 +Ethanol + GT

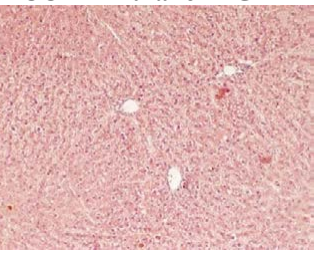

F

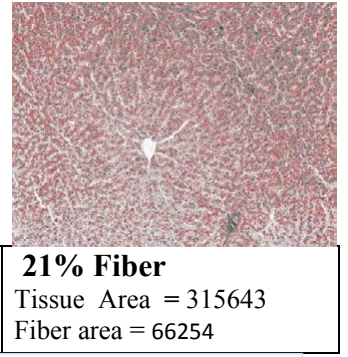

Fiber area $=66254$

Figure 2: Quantification of Fiber comparison with different group.

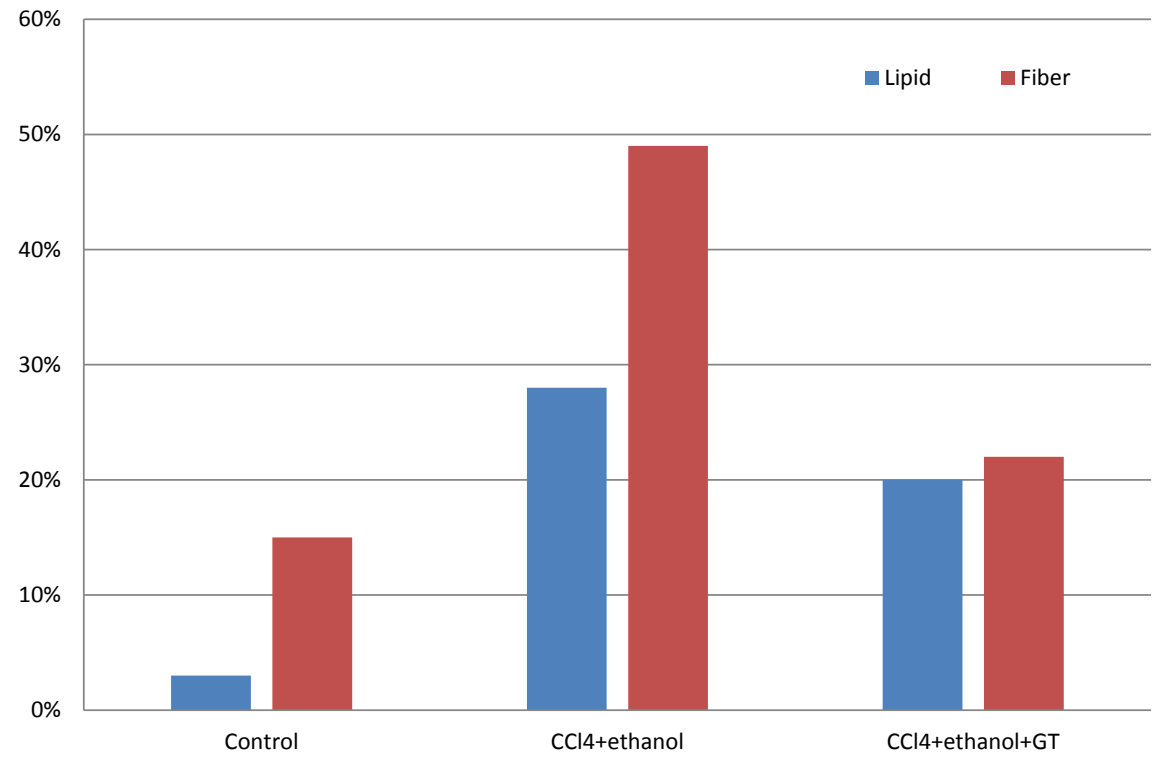

Figure 3: Quantification Percentage of fiber in different group.

Fiber Prcentage

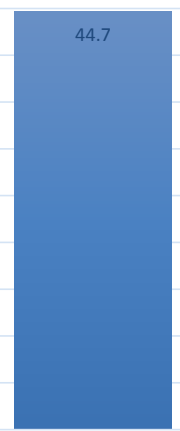

ccl4 eth

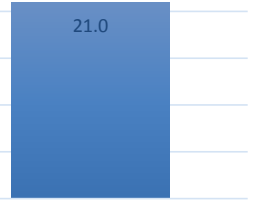

ccl4 eth GT 
Citation: Safer AM (2017) A Quantitative Description of Lipid and Extracellular Matrix Proteinaceous Fibers in Hepatic Fibrosis of a Rat Model by Image J using Nano-Images. J Nanomed Nanotechnol 8: 446. doi: 10.4172/2157-7439.1000446

the effect of $\mathrm{CCl}_{4}$ and ethanol became visible bright red in color (Figure $1 \mathrm{~b})$. The total percentage of lipid was $28 \%$. Comparing to the one treated with nanogreen tea extract in the presence of $\mathrm{CCl}_{4}$ and ethanol $20 \%$ (Figure 1c). As indicated in the images of the control, the lipid in the damaged is was only $3 \%$ from the total non-lipid area $13117876 \mathrm{~nm}^{2}$, while the lipid area was $100749 \mathrm{~nm}^{2}$. In the completely damaged group, the total lipid was as high as $28 \%$, where the non-lipid area was 3145720 $\mathrm{nm}^{2}$ versus the lipid area $768008 \mathrm{~nm}^{2}$. In the third group of ethanol/ $\mathrm{CCl}_{4}$ and nanoGTE, the total lipid are came down to $20 \%$, where the non-lipid area was $3145728 \mathrm{~nm}^{2}$ and the lipid area was $1219178 \mathrm{~nm}^{2}$. $[5,10]$.

\section{Protein fiber analysis}

Once threshold was set at a proper scale from normal control (Figure 2a), all fibers in the damaged tissue under study became visible bright red in colour (Figure $2 \mathrm{~b}$ ) with total percentage of fibers $49 \%$. Comparing to the one treated with nanogreen tea extract in the presence of $\mathrm{CCl}_{4}$ and ethanol 22\% (Figure 2c). As indicated in the images of the control, the fiber in the damaged was only $15.9 \%$ from the total non-fiber area, In the completely damaged group the total fiber was as high as $44.7 \%$. In the third group of ethanol/ $\mathrm{CCl}_{4}$ and nanoGTE the total fiber area came down to $21 \%$, The two parameters for the three groups of rat are clear in the histogram where the big difference between the damaged case when ethanol/ $/ \mathrm{CCl}_{4}$ are used for three weeks to create fibrosis and the ethanol/ $\mathrm{CCl}_{4}$ and nanoGTE are used as antifibrotic therapeutic agent (Figure 2) [4]. The effect of nanogreen tea extract is clear in the tested group in comparison to the control group and the ethanol. $\mathrm{CCl}_{4}$ group as shown in the two histograms provided (Figures 2 and 3 ). When nano SEM images were resolved and analyzed, It was found that there are two distinc types of fibers formed in the ECM during fibrosis stages; one due to the effect of $\mathrm{CCl}_{4}$ (Figure 4A) while the other was due to

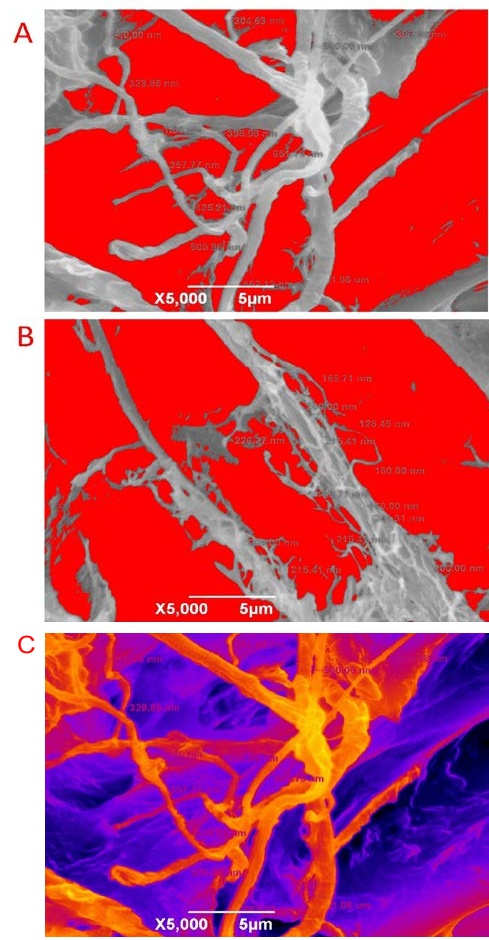

Figure 4: Showing fibrous proteins in the ECM of rat fibrotic hepatocyte with specified pattern. A. shows fibers formed due to the effect of $\mathrm{CCl} 4$, B. shows fibers formed due to the effect of ethano. C. shows image of combined fibers. X5000. ethanol (Figure 4B). A combined pattern of fibers were also discerned as in (Figure 4C). The ECM fibers were completely eliminated from the scene after three weeks of nanogreen tea treatment.

\section{Discussion}

Here we analyzed the previously published work on the effects of nanogreen tea extract on hepatic fibrosis using various histological and histochemical techniques and electron microscopy analyses; both TEM and SEM [11,12] by using the ImageJ software. This piece of work is aimed to confirm the therapeutic effects of nanogreen tea extract on hepatic fibrosis and how it reverses tissue structure and cell organelles to their normal norm and how it eradicates ECM fibers all over the place. This technique provides quantitative descriptions of the fiber arrays and their average orientation in ECM $[13,14]$, directly from the histologic stained images on one hand and the nano images obtained from high-quality TEM and SEM images on the other. With these software combined, fibrillar structures, such as collagen, or the texture of various materials have been resolved quantitatively. The tool provides the location and quantification of both lipid droplets (steatosis) and the ECM fibrous proteins in the fibrotic liver before and after the treatment with nano-green tea extract and the average orientation and anisotropy of fiber arrays in a given region of interest $[10,12]$.

As for Lipid Analysis, once the threshold was set at a proper scale, from normal control all lipid droplets in the damaged tissue under study due to the effect of $\mathrm{CCl}_{4}$ and ethanol became visible bright red in color, with the total percentage of lipid was $28 \%$. Comparing to the one treated with nanogreen tea extract in the presence of $\mathrm{CCl}_{4}$ and ethanol $20 \%$ and with reference to the control group of zero damage. The total damaged are in control was found as 100749 and mean 153.00 while in $\mathrm{CCl}_{4} /$ ethanol group it was 314540 and mean 114.00 in $\mathrm{CCl}_{4} /$ ethanol/ CCl4/ethanol/NanoGTE was 3145728 with mean 129.00 [5].

For protein fiber analysis; once the threshold was set at a proper scale, the damaged tissue under study became visible bright red in colour with the total percentage of fibers $49 \%$. Comparing to the one treated with nanogreen tea extract in the presence of $\mathrm{CCl}_{4}$ and ethanol $22 \%$ and the zero in control. The total damaged are in control was found as $15.9 \%$ and in $\mathrm{CCl}_{4}$ /ethanol group it was $44.7 \%$ and in $\mathrm{CCl}_{4} /$ ethanol/Nano GTE was $21 \%[5,9,12,15]$.

The two parameters for the three groups of the rat are clear in the histogram where apart from the control, it clearly shows the big difference between the damaged case when ethanol/ $\mathrm{CCl}_{4}$ are used for three weeks to create fibrosis and the ethanol/ $\mathrm{CCl}_{4}$ and nanoGTE are used as the antifibrotic therapeutic agent (5.11).

Once again imageJ technique confirms what was seen in previous work $[11,12,16]$ that nanoGTE when used for rat hepatic fibrosis model treatment for a period of three weeks, will eradicate all ECM fibrous protein and most of the lipid in the cell cytoplasm almost to its normal norm [5]. When ECM fibers were resolved and examined with high quality SEM nanoimages,two distinct types of fibers were clearly visible; one was formed due to $\mathrm{CCl} 4$ and the other was formed due to ethanol. These fibers are native to the ECM of various tissues specifically accumulated when tiises are under abnormal or diseased condition $[17,18]$.

\section{Acknowledgments}

The author wishes to thank Mr. Nemany Hanafy from the Department of Biological Sciences, Ms. Ahlam AlKadi and The author acknowledge assistance offered by Ayman E El-Sharkawey for imageJ analysis, from the Nanoscopy Science Center, Faculty of Science, Kuwait University. 
Citation: Safer AM (2017) A Quantitative Description of Lipid and Extracellular Matrix Proteinaceous Fibers in Hepatic Fibrosis of a Rat Model by ImageJ using Nano-Images. J Nanomed Nanotechnol 8: 446. doi: 10.4172/2157-7439.1000446

\section{References}

1. Taura K, Miura K, Iwaisako K, Osterreicher CH, Kodama Y, et al. (2010) Hepatocytes do not undergo epithelial-mesenchymal transition in liver fibrosis in mice. Hepatology 51: 1027-1036.

2. Ehrhardt N, Doche ME, Chen S, Mao HZ, Walsh MT, et al. (2017) Hepatic Tm6sf2 overexpression affects cellular ApoB-trafficking, plasma lipid levels, hepatic steatosis and atherosclerosis. Hum Mol Genet.

3. Jarido V, Kennedy L, Hargrove L, Demieville J, Thomson J, et al. (2017) The emerging role of mast cells in liver disease. American Journal of Physiology Gastrointestinal and Liver Physiology.

4. Safer AM, Hanafi N, Bharali DJ (2015) Effect of Green Tea Extract Encapsulated Into ChitosanNanoparticles on Hepatic Fibrosis Collagen FibersAssessed by Atomic Force Microscopy in Rat Model Hepatic Fibrosis. Journal of Nanoscience and Nanotechnology 15: 6452-6459.

5. Safer AM (2017) Remediation of hepatic fibrosis as a result of the use of $\mathrm{CCl} 4$ and Ethanol by Chitosan Nano-Green Tea Extract: Quantification and Ultrastructural Studies. Journal of Nanomedicine Research, J Nanomed Res 5: 00127.

6. Rasband WS (2014) ImageJ US National Institutes of Health, USA.

7. Schneider CA, Rasband WS, Eliceiri KW (2012) NIH Image to ImageJ: 25 years of image analysis. Nature methods $671-675$.

8. Abramoff MD, Magalhaes PJ, Ram SJ (2004) Image Processing with ImageJ. Biophotonics International.

9. Bruno RS, Dugan CE, Smyth JA, DiNatale DA, Koo SI (2008) Green tea extract protects leptin-deficient, spontaneously obese mice from hepatic steatosis and injury. J Nutr 138: 323-331.

10. Murase T, Haramizu S, Shimotoyodome A, Tokimitsu I, Hase T (2006) Green tea extract improves running endurance in mice by stimulating lipid utilization during exercise. Am J Physiol Regul Integr Comp Physiol 290: R1550-R1556.
11. Safer A, Sen A, Hanafy NA, Mousa SA (2015) Quantification of the Healing Effect in Hepatic FibrosisInduced by Chitosan Nano-EncapsulatedGreen Tea in Rat Model. Journal of Nanoscience and Nanotechnology 15: 9918-9924.

12. Safer AM, Afzal M, Hanafy N, Mousa S (2014) Green tea extract therapy diminishes hepatic fibrosis mediated by dual exposure to carbon tetrachloride and ethanol: A histopathological study. Experimental and therapeutic medicine 9: 787-794.

13. Liu Z, Pouli D, Sood D, Sundarakrishnan A, Hui Mingalone CK, et al. (2016) Automated quantification of three-dimensional organization of fiber-like structures in biological tissues. Biomaterials 116: 34-47.

14. Sivaguru M, Kabir MM, Gartia MR, Biggs DSC, Sivaguru BS, et al. (2017) Application of an advanced maximum likelihood estimation restoration method for enhanced-resolution and contrast in second-harmonic generation microscopy. J Microsc.

15. Park HJ, DiNatale DA, Chung MY, Park YK, Lee JY, et al. (2011) Green tea extract attenuates hepatic steatosis by decreasing adipose lipogenesis and enhancing hepatic antioxidant defenses in ob/ob mice. J Nutr Biochem 22 393-400.

16. Afzal M, Safer AM, Menon M (2015) Green tea polyphenols and their potentia role in health and disease. Inflammopharmacology 23: 151-161.

17. Klaas M, Kangur T, Viil J, Mäemets-Allas K, Minajeva A, Vadi K, Antsov M, Lapidus $N$, Järvekülg $M$, Jaks The alterations in the extracellular matrix composition guide the repair of damaged liver tissue. Sci Rep. 2016; 6: 27398

18. Yutaka Yasui, Tokiya Abe, Masayuki Kurosaki, Mayu Higuchi, Yasuyuki Komiyama, Tsubasa Yoshida, Tsuguru Hayashi, Konomi Kuwabara, Kenta Takaura, Natsuko Nakakuki, Hitomi Takada, Nobuharu Tamaki, Shoko Suzuki, Hiroyuki Nakanishi, Kaoru Tsuchiya, Jun Itakura, Yuka Takahashi, Akinori Hashiguchi, Michiie Sakamoto, and Namiki Izumi. Elastin Fiber Accumulation in Liver Correlates with the Development of Hepatocellular Carcinoma. PLoS One. 2016; 11(4): e0154558. 